\title{
Associations between fatty acid intakes and plasma phospholipid fatty acid concentrations in the European Prospective Investigation into Cancer and Nutrition
}

\section{Abstract \\ Introduction}

Fatty acids (FA) are highly active molecules involved in different metabolic pathways. Several FA have direct tumorigenic effects in animal and cell-line models and recent epidemiological studies also suggest associations with cancer risk. Some of these health effects are associated with the phospholipid (PL) FA composition of cell membranes what may be related to dietary FA intake. This study aims to assess the correlation between FA intake and the plasma PL FA status in the European Prospective Investigation into Cancer and Nutrition (EPIC) cohort.

\section{Methods}

Dietary intake of individual FA was estimated using centre-specific validated dietary questionnaires and the National Nutrient Database for Standard Reference of the United States. Circulating levels of 60 individual PL FA were measured in baseline venous plasma phospholipid samples in nested case-controls studies within the EPIC cohort $(n=9,996)$. The fatty acid composition in the phospholipid fraction was measured by gas chromatography in plasma samples. Spearman rank correlations were calculated to determine associations between FA intakes and plasma PL levels.

\section{Results}

Results indicated low to moderately high associations between FA intake and plasma levels. Low correlations were found between intakes of total saturated FA or total monounsaturated FA and plasma levels. Moderate positive correlations were particularly found for long-chain $n-3$ poly-unsaturated FA $(r=0.35)$ with the highest $(r=0.41)$ for $n-3$ PUFA docosahexaenoic acid (DHA). Moderately high correlations were found for the exogenous trans-FA ( $\mathrm{r}=0.53$ for total trans-FA; $r=0.48$ for industrial trans-FA (elaidic acid)). Sensitivity analysis showed some attenuation of most correlations among cancer cases compared to controls.

\section{Discussion}

Our findings suggest that dietary FA intake influences the plasma PL FA status to a certain extent for several FA isomers, particularly those that are not or less efficiently endogenously produced. As diet is a modifiable risk factor, these findings are important for future public health strategies focusing on cancer prevention. Although the level of detail in dietary questionnaires is rather limited, these results are showing their potential to assess the intake of FA isomers in large-scale populations where biological measurements are not feasible for the full cohort.

\section{Conflict of Interest}

There is no conflict of interest 